Military Technical College Kobry El-Kobba Cairo, Egypt

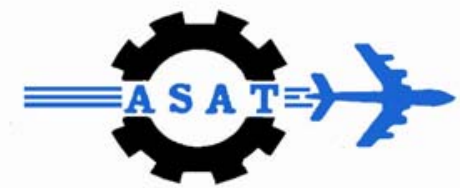

12-th International Conference

on

Aerospace Sciences \&

Aviation Technology

\title{
A SIMPLIFIED METHOD TO EVALUATE MEAN EFFECTIVE GAIN OF MOBILE ANTENNAS
}

\author{
Mohamed A.H. Eleiwa*
}

\begin{abstract}
A procedure, based on analytical formulations, and antenna pattern cuts in different planes, is introduced for evaluating and analyzing the mobile antenna effective gain $\left(\mathbf{G}_{\mathbf{e}}\right)$ in multipath propagation environments. First, analytical expressions, which are relevant for numerical calculations of $\boldsymbol{G}_{e}$, are derived. Then, different multipath propagation environments are modeled using statistical distributions for angular density functions of the randomly incident waves. The proposed procedure is finally applied to evaluate the mean effective gain (MEG) of the reference $\mathbf{N} \mathbf{2}$ and short dipole antennas in different mobile environments. Also, an inverted-L mobile handheld antenna is properly designed for GSM900 cellular system, and its MEG characteristics are investigated graphically using different co-polar and cross-polar antenna pattern cuts in different planes.
\end{abstract}

\section{KEY WORDS}

Mobile communications, Antennas, Effective gain

\section{INTRODUCTION}


The performance of a mobile antenna is strongly dependent on its effective gain $\left(\boldsymbol{G}_{\mathrm{e}}\right)$, or alternatively known as mean effective gain $(\boldsymbol{M E G})$ in a multipath propagation environment [1]. Since the gain influences the size of the radio zone, transmitting power of mobile radio equipment, and, in particular, battery capacity in the case of portable radio equipment. Furthermore, in an antenna diversity system, the diversity gain is determined not only by correlation characteristics between signal envelopes received in the antenna elements, but also by the effective gain characteristics of antenna elements. Therefore, for designing a mobile antenna system, it is essential to investigate mobile antenna $\boldsymbol{G}_{e}$ characteristics and to develop the techniques to maximize the effective gain for the anticipated mobile radio environment.

However, it is a common knowledge that the $\boldsymbol{G}_{\mathrm{e}}$ of mobile antennas in land mobile propagation environments cannot be evaluated with sufficient accuracy from just antenna directive gain, since the received signals undergo Rayleigh-like fading, and random multipaths exist due to reflection, diffraction, and scattering. Hence, the experimental method [2] is used to measure the mean signal level received over a certain route for evaluating the effective gain. With this method, the mean power levels of the unknown antenna and a reference antenna ( $\lambda / 2)$ are obtained by averaging the signal levels received while each antenna moves along the same selected route. This method is useful for measuring the $G_{e}$ of mobile antennas in practical environments, and it has been used for evaluating the $G_{e}$ of several mobile antennas [2-3]. However, measured $\boldsymbol{G}_{\boldsymbol{e}}$ reflects the interaction between the antenna power gain pattern and the propagation characteristics along the route; it depends on the measurement route. Even though the reference antenna also experiences the same interaction effects, the conventional experimental method is not accurate enough to evaluate the $G_{e}$ of mobile antennas in general. Therefore, a simplified method is introduced to determine the $\boldsymbol{G}_{e}$ of mobile antennas in multipath propagation environments.

The proposed method for $G_{e}$ evaluation is based on the joint contribution of the antenna patterns and the statistical distribution of the propagating waves. The analytical expressions for $\boldsymbol{G}_{\boldsymbol{e}}$ formulas are firstly derived for mobile antennas in different propagation environments, which are defined by the angular density statistical distribution wave functions. Numerical routines are then developed to calculate $\boldsymbol{G}_{\mathrm{e}}$ for different mobile antennas in different multipath propagation environments, and the optimum conditions to yield maximum $G_{e}$ are then drawn. Moreover, a graphical technique is introduced to estimate $\boldsymbol{G}_{\mathrm{e}}$, where $\boldsymbol{G}_{\mathrm{e}}$ estimation may result from careful reading of different co-polar and cross-polar antenna pattern cuts in different planes. Finally, an inverted-L antenna is designed for mobile handheld devices, and proper matching is achieved within the frequency range of GSM900 cellular system. Different co-polar and cross-polar pattern cuts are plotted in different planes, from which, the cross polarization $X P R$ values are determined, and hence $\boldsymbol{G}_{e}$ characteristics may be predicted.

\section{MEG EVALUATION TECHNIQUES}

The ratio between the mean received power of antenna over the random route $\boldsymbol{P}_{\text {rec }}$ and the total mean arriving power $\boldsymbol{P}_{\boldsymbol{V}}+\boldsymbol{P}_{\boldsymbol{H}}$ can be considered as the $\boldsymbol{G}_{\boldsymbol{e}}$ of the mobile 
antenna in the environment. It is assumed that the average power over a random route in an environment equals the average over the environment. $\boldsymbol{G}_{\mathrm{e}}$ is given by [4] as

$$
G_{e}=\frac{P_{r e c}}{P_{V}+P_{H}}
$$

where $\boldsymbol{P}_{\boldsymbol{V}}$ and $\boldsymbol{P}_{\boldsymbol{H}}$ are, respectively, the mea powers of the vertically polarized (VP) and horizontally polarized $(\boldsymbol{H P})$ radio waves received while the antenna moves over a random route in the environment. First it is necessary to establish a theoretical $\boldsymbol{G}_{\boldsymbol{e}}$ expression that takes into account the $\boldsymbol{V P}$ and $\boldsymbol{H P}$ radio waves in multipath environments.

The mean power ratio of the incident $V \boldsymbol{P}$ and $\boldsymbol{H P}$ radio waves is defined as the cross-polarization power ratio $(\boldsymbol{X P R})$ :

$$
X P R=\frac{P_{V}}{P_{H}}
$$

$X P R$ corresponds to the cross-polarization coupling when the polarization of the transmitted radio waves is horizontal, and to the reciprocal of the cross-polarization coupling when the polarization of transmitted waves is vertical. In a spherical coordinate system, the mean antenna received power $\boldsymbol{P}_{\text {rec }}$ is expressed by the following [6]:

$$
P_{r e c}=\int_{0}^{2 \pi} \int_{0}^{\pi}\left[P_{1} G_{\theta}(\theta, \phi) P_{\theta}(\theta, \phi)+P_{2} G_{\phi}(\theta, \phi) P_{\phi}(\theta, \phi)\right] \sin \theta d \theta d \phi
$$

where $\boldsymbol{P}_{\boldsymbol{1}}$ is the mean power that would be received by a $\hat{\boldsymbol{\theta}}$-polarized isotropic antenna in the mobile radio environment. Similarly, $\boldsymbol{P}_{\mathbf{2}}$ is the mean power that would be received by a $\hat{\phi}$-polarized isotropic antenna, $G_{\theta}(\theta, \phi)$ and $G_{\phi}(\theta, \phi)$ are the $\boldsymbol{\theta}$ and $\boldsymbol{\Phi}$ components of the antenna power gain pattern, $\boldsymbol{P}_{\boldsymbol{\theta}}(\boldsymbol{\theta}, \boldsymbol{\phi})$ and $P_{\phi}(\theta, \phi)$ are the $\theta$ and $\Phi$ components of the angular density functions of incoming plane waves; respectively. These functions satisfy the following conditions:

$$
\begin{aligned}
& \int_{0}^{2 \pi} \int_{0}^{\pi}\left[G_{\theta}(\theta, \phi)+G_{\phi}(\theta, \phi)\right] \sin \theta d \theta d \phi=4 \pi \\
& \int_{0}^{2 \pi} \int_{0}^{\pi}\left[P_{\theta}(\theta, \phi)\right] \sin \theta d \theta d \phi=1 \\
& \int_{0}^{2 \pi} \int_{0}^{\pi}\left[P_{\phi}(\theta, \phi)\right] \sin \theta d \theta d \phi=1
\end{aligned}
$$

Assuming the mobile antenna moves in the $X-Y$ plane, the $\boldsymbol{\theta}$ and $\boldsymbol{\Phi}$ components correspond to the $\boldsymbol{V P}$ and $\boldsymbol{H P}$ components. Thus the terms $\boldsymbol{P}_{\mathbf{1}}$ and $\boldsymbol{P}_{\mathbf{2}}$ are the mean received power of $\boldsymbol{V P}$ and $\boldsymbol{H P}$ isotropic antennas; respectively, and $\boldsymbol{X P R}$ is equal to the ratio $\boldsymbol{P}_{\mathbf{1}} / \boldsymbol{P}_{\mathbf{2}}$. By using (3) and the $\boldsymbol{X P R}$ notation, the expression for MEG can be rearranged to yield the following equation 
$G_{e}=\int_{0}^{2 \pi n} \int_{0}\left[\frac{X P R}{1+X P R} G_{\theta}(\theta, \phi) P_{\theta}(\theta, \phi)+\frac{1}{1+X P R} G_{\phi}(\theta, \phi) P_{\phi}(\theta, \phi)\right] \sin \theta d \theta d \phi$

When only a VP wave is incoming from a single $\left(\theta_{S}, \phi_{S}\right)$ direction, which corresponds to line of sight propagation with $V P$ wave transmission, the angular density functions are represented as $P_{\theta}(\theta, \phi)=\frac{\delta\left(\theta-\theta_{S}\right) \cdot \delta\left(\phi-\phi_{S}\right)}{\sin \theta_{S}}$, and $\boldsymbol{P}_{\phi}(\theta, \phi)=0$. It then follows from equation (7) that MEG becomes

$G_{e}=G_{\theta}\left(\theta_{s}, \phi_{s}\right)$

This means that $G_{e}$ corresponds to the antenna directive gain in the $\left(\theta_{S}, \phi_{S}\right)$ direction when incoming signals are centered on the $\left(\boldsymbol{\theta}_{\boldsymbol{S}}, \boldsymbol{\phi}_{S}\right)$ direction.

If the characteristics of incoming signals in various environments can be represented as statistical distribution functions $\boldsymbol{P}_{\boldsymbol{\theta}}, \boldsymbol{P}_{\boldsymbol{\phi}}$, the $\boldsymbol{G}_{\boldsymbol{e}}$ given in (7) is the mean power gain of the antenna in each environment.

If the incident waves arriving at the mobile antenna are concentrated in the horizontal plane [39], and that the angular density functions of the incident waves are given by:

$$
P_{\theta}=P_{\phi}=\frac{1}{2 \pi} \delta\left(\theta-\frac{\pi}{2}\right)
$$

Substituting (9) into (7) gives the following simplified MEG form as

$$
G_{e}=\frac{1}{2 \pi} \int_{0}^{2 \pi}\left[\frac{X P R}{1+X P R} G_{\theta}\left(\frac{\pi}{2}, \phi\right)+\frac{1}{1+X P R} G_{\phi}\left(\frac{\pi}{2}, \phi\right)\right] d \phi
$$

It is quite reasonable to adopt a statistical model in which angular density functions $\boldsymbol{P}_{\boldsymbol{\theta}}, \boldsymbol{P}_{\boldsymbol{\phi}}$ are assumed to be Gaussian in elevation and uniform in the azimuth direction [6], noting that the elevation can take on negative values, since mobile antennas are usually operated above the ground. The distribution functions of incident plane waves are expressed as follows

$$
\begin{array}{ll}
\boldsymbol{P}_{\theta}(\theta, \phi)=A_{\theta} \exp \left\{-\frac{\left[\theta-\left(\frac{\pi}{2}-m_{v}\right)\right]^{2}}{2 \sigma_{V}^{2}}\right\}, & 0 \leq \theta \leq \pi \\
\boldsymbol{P}_{\phi}(\theta, \phi)=A_{\phi} \exp \left\{-\frac{\left[\theta-\left(\frac{\pi}{2}-m_{H}\right)\right]^{2}}{2 \sigma_{H}^{2}}\right\}, & 0 \leq \theta \leq \pi
\end{array}
$$

where $\boldsymbol{m}_{\boldsymbol{v}}$ and $\boldsymbol{m}_{\boldsymbol{H}}$ are the mean elevation angle of each VP and HP wave distribution observed from the horizontal direction, and $\sigma_{V}$ and $\sigma_{H}$ are the standard deviation of each VP and $\boldsymbol{H P}$ wave distribution. $\boldsymbol{A}_{\boldsymbol{\theta}}$ and $\boldsymbol{A}_{\phi}$ are constants determined by substituting (11) and (12) into (5) and (6) to yield 


$$
\begin{aligned}
& A_{\theta}=\frac{1}{2 \pi \int_{0}^{\pi} \exp \left\{-\frac{\left[\theta-\left(\frac{\pi}{2}-m_{V}\right)\right]^{2}}{2 \sigma_{V}^{2}}\right\} \sin \theta d \theta} \\
& A_{\phi}=\frac{1}{2 \pi \int_{0}^{\pi} \exp \left\{-\frac{\left[\theta-\left(\frac{\pi}{2}-m_{H}\right)\right]^{2}}{2 \sigma_{H}^{2}}\right\} \sin \theta d \theta}
\end{aligned}
$$

\section{ESTIMATION OF MEG FOR MOBILE ANTENNAS}

A numerical algorithm is developed to calculate $\boldsymbol{G}_{\boldsymbol{e}}$ for mobile antennas in multipath propagation environments. First, the mobile communication environments are generally modeled by statistical (Gaussian) distributions of the angular density functions as given by equations (11) and (12). Such angular density functions are then substituted into equation (7) to yield the mobile antenna effective gain. Intensive calculations have been performed to investigate MEG variation for $\mathbf{N} / \mathbf{2}$ and short dipoles as function in the mobile environment represented by standard deviations $\sigma_{V}$ and $\sigma_{H}$, and the mean elevation angle $\boldsymbol{m}_{\boldsymbol{v}}$ and $\boldsymbol{m}_{\boldsymbol{H}}$, and the results are plotted in Figs.1, 2, 3 and 4. MEG for $\boldsymbol{N} / \mathbf{2}$ and short dipoles are compared for $\boldsymbol{m}_{v}=25^{\circ}$, and different $\sigma_{V}$ from $0^{\circ}$ to $90^{\circ}$ in Fig.1, and for $\sigma_{v}=27^{\circ}$, and different $\boldsymbol{m}_{v}$ from $0^{\circ}$ to $90^{\circ}$ in Fig.2. The degradation in dipoles MEG due to multipath environment parameters $\left(\sigma_{v}\right.$ and $\left.\boldsymbol{m}_{v}\right)$ is very clear in both figures, where maximum MEG for $\mathbf{N} \mathbf{2}$ and short dipoles are found from Fig. 1 to be $0.92 \mathrm{~dB}$ and $0.9 \mathrm{~dB}$; respectively, compared to their directive gains $2.15 \mathrm{~dB}$ and $1.8 \mathrm{~dB}$; respectively. Similar results are shown in Fig.2. The variation of MEG for $\mathbf{N} / \mathbf{2}$ and short dipoles are also investigated at different statistical parameters as shown in Figs.3 and 4; respectively. From these figures, it worthy noticed that there are some propagation parameters that yield optimum MEG, and also constant MEG may be obtained regardless of the environmental parameters variations. Alternatively, there is a possibility of tailoring an artificial propagation environment, in which MEG is optimum and constant regardless of the antenna pattern and polarization variations. Such artificial propagation environment may be developed by controlling the polarity, height, and beam width of the transmitting antenna to adjust XPR, mean elevation angle, and the standard deviation; respectively. But further theoretical and experimental investigations are required to confirm this. 


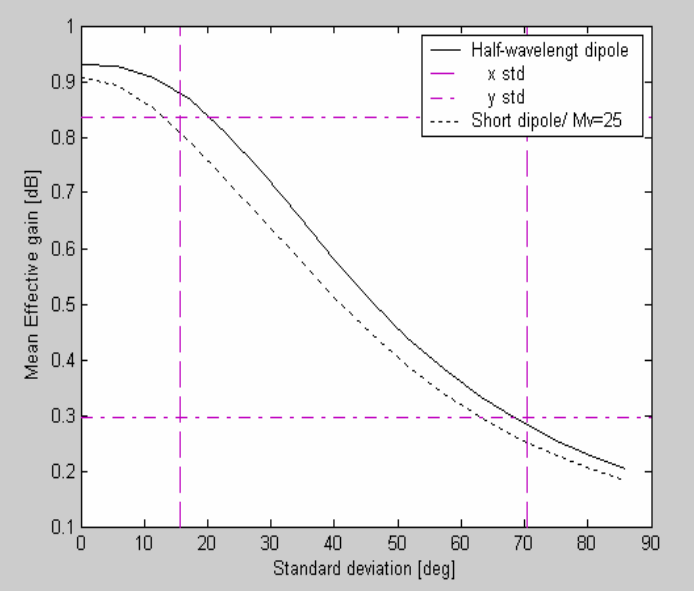

Fig. 1. Dipoles MEG variation vs. $\sigma_{v}$.

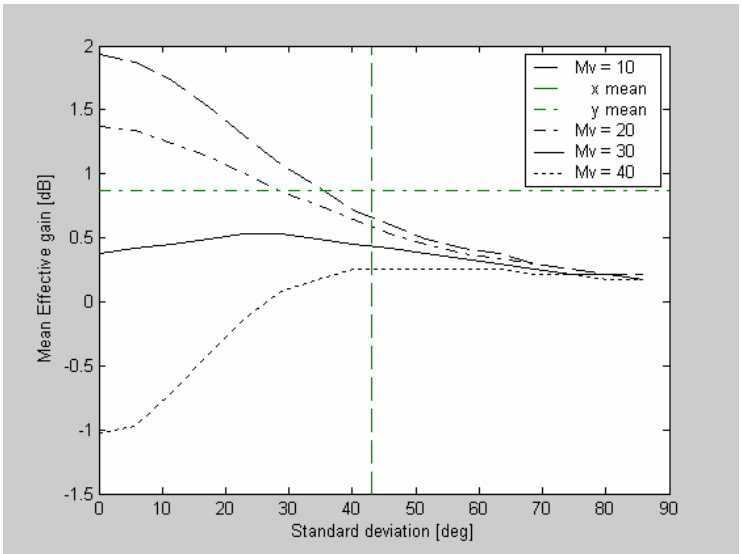

Fig. 3. MEG of vertically oriented $\lambda / 2$ dipole antenna for vertically polarized incident waves with different statistical parameters

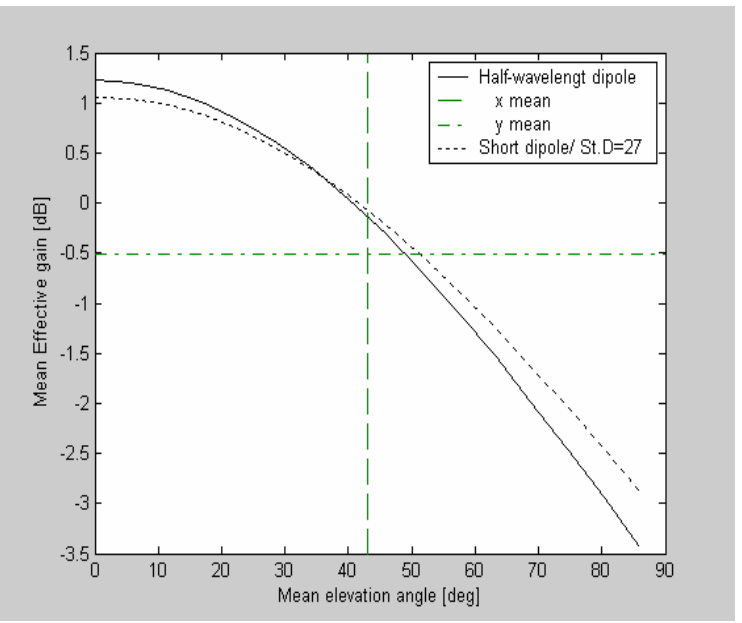

Fig. 2. Dipoles MEG variation vs. $\boldsymbol{m}_{\boldsymbol{v}}$.

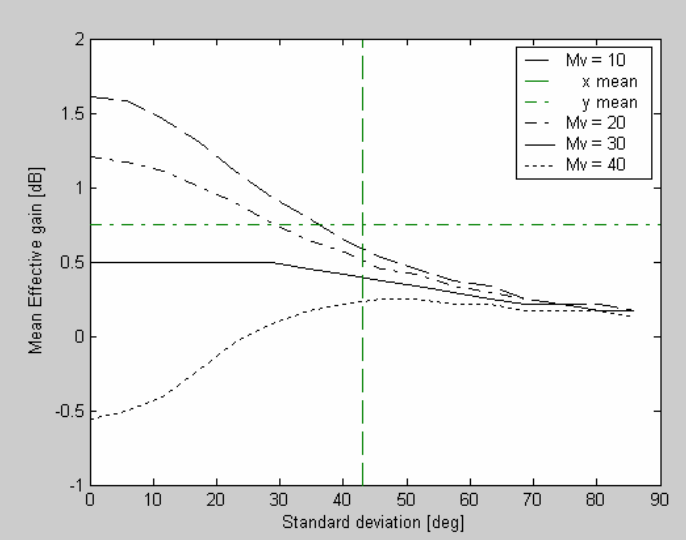

Fig. 4. MEG of vertically oriented short dipole antenna for vertically polarized incident waves with different statistical parameters

A graphical procedure is also proposed to estimate MEG mobile antennas. The proposed procedure is applied to estimate MEG characteristics of an inverted-L antenna designed for GSM900 cellular system. The inverted-L antenna on the handset case (conducting box), as shown in Fig. 5, is modeled using WIPL-D software package. The box dimensions and wire parameters are adjusted to yield proper matching ( $V S W R<2.23$ ) within the frequency range of GSM900, as shown in Fig. 6. Different co-polar and cross-polar antenna pattern cuts at different frequencies are then plotted as shown in Fig.7 a, b, c and d. In these figures, the XPR is first calculated, and hence MEG at a certain direction by comparing the values of co-polar and cross-polar gain components. The XPR off-bore sight is also known as the polarization quality factor (PQR). 


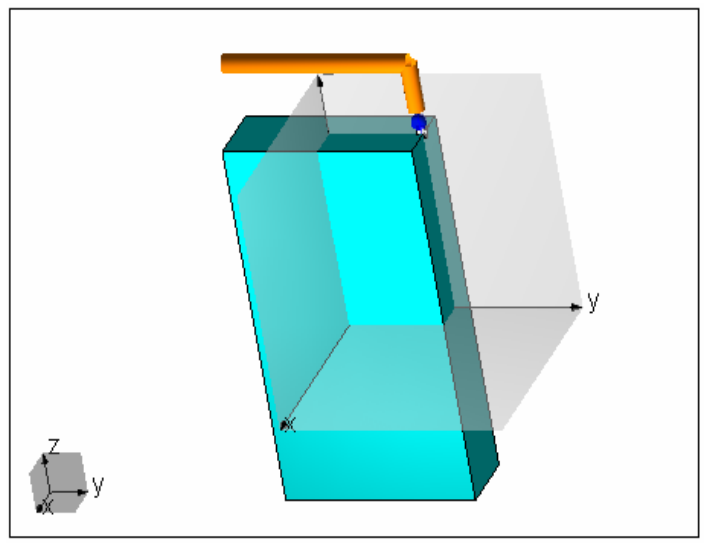

Fig. 5. Inverted-L mobile antenna

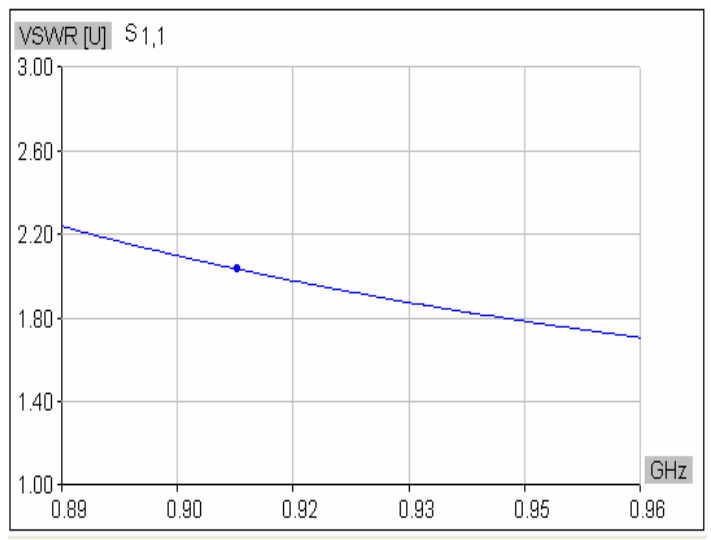

Fig. 6. VSWR for Inverted-L mobile antenna vs. frequency.

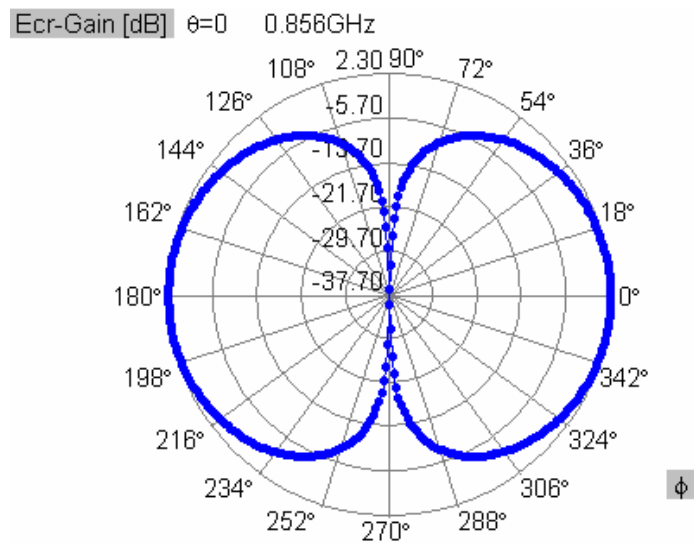

(b)

Fig.7(a, b). Different co-polar and cross-polar pattern cuts for inverted-L antenna

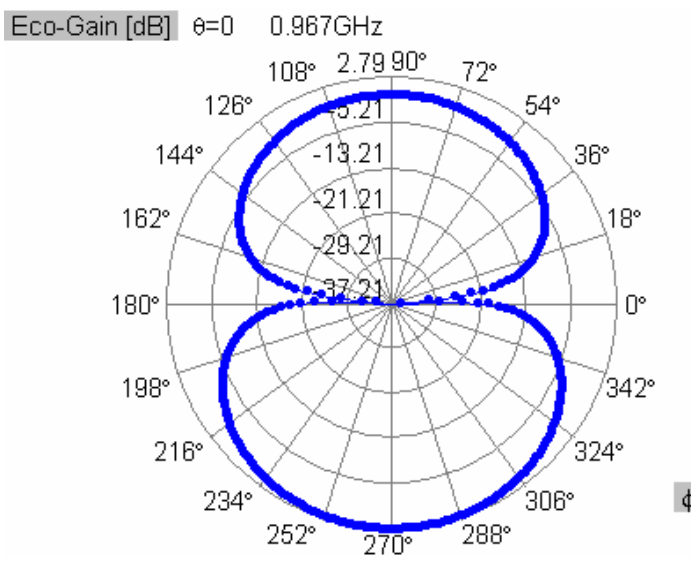

(c)

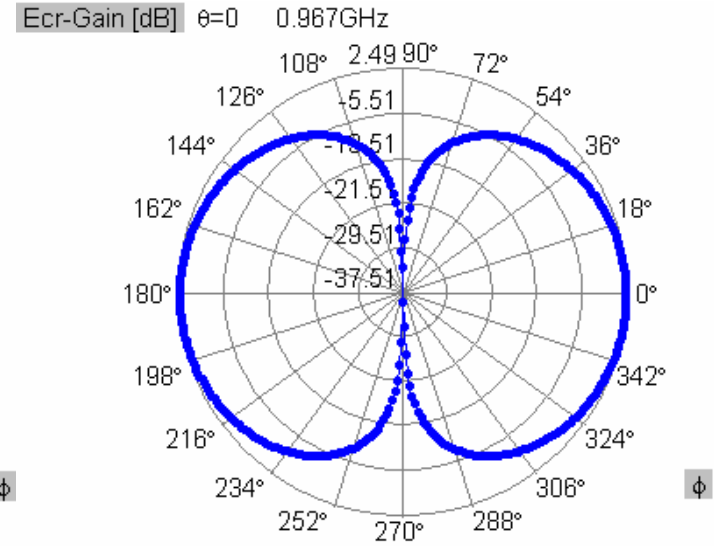

(d)

Fig. 7(c,d). Different co-polar and cross-polar pattern cuts for inverted-L antenna

\section{CONCLUSIONS}


Different approaches have been developed for evaluation of mobile antennas effective gain. The proposed procedure is based on two approaches: the numerical calculations of relevantly derived MEG expressions, and the antenna pattern plane cuts for co-polar and cross-polar components. The proposed procedure has been applied to investigate numerically the MEG for $\mathbf{N} \mathbf{2}$ and short dipole antennas in different multipath propagation environments, which have been modeled by statistical distributions of the angular density functions for the randomly arriving waves from such environments. The intensive numerical calculations revealed the possibility of developing an artificial propagation environment, in which MEG is maximum and constant regardless of the variation of the antenna pattern and polarization. Finally, an inverted-L antenna was optimally designed for GSM900 cellular system, and its MEG characteristics have been estimated from different co-polar and cross-polar pattern cuts.

\section{REFERENCES}

[1] P. Krishnamurthy Pahlavan, K. and Krishnamurthy, P. Krishnamurthy, "Principles of Wireless Networks-A Unified Approach." Englewood Cliffs, NJ: Prentice-Hall, 2002.

[2] Andersen, Bach, J., and Hansen, F. "Antennas for VHF/UHF Personal Radio: A Theoretical and Experimental Study of Characteristics and Performance," IEEE Trans. Vehicular Technology, Vol. VT-26, No.4, Nov. 1977, pp. 349-357.

[3] Davidson, A. L., and Turney,W.J. "Mobile Antenna Gain in Multipath Environment at $900 \mathrm{MHz}$," IEEE Trans. Vehicular Technology, Vol. VT-26, No.4, Nov. 1977, pp. 345-348.

[4] Taga, T., "Analysis for Mean Effective Gain of Mobile Antennas in Land Mobile Radio Environments," IEEE Trans. Vehicular Technology, Vol. VT-39, No.2, May 1990, pp. 117-131.

[5] Cox, D.C., R.R. Murray, Arnold, H.W. Norris, A.W. and Wazowicz, M.F. "CrossPolarization Coupling Measured for $800 \mathrm{MHz}$ Radio Transmission in and Around Houses and Large Buildings," IEEE Trans. Ant. Propag., Vol. AP-34, No. 1, Jan. 1986, pp. 83-87.

[6] Lee, W.C.Y., "Mobile Communication Engineering," McGraw-Hill, 1982. 